\title{
Ein pathogenetischer Faktor
}

Fragestellung: Diese Studie untersucht, inwieweit eine Infektion mit dem Varizella-Zoster-Virus (VZV) bei Patienten mit Verdacht auf eine Arteriitis temporalis (Riesenzellarteriitis, RZA) ohne histologischen Nachweis einer RZA in der Biopsie vorhanden ist.

Hintergrund: Bei Patienten mit Verdacht auf eine RZA, die dann aber in der Biopsie histologisch nicht bestätigt werden konnte, wurden Infektionen mit VZV nachgewiesen [1]. Zudem wurden bei biopsiepositiven RZA in $74 \%$ der Biopsien VZV gefunden [2]. Beides legt eine Rolle von VZV in der Pathogenese der Arteriitis temporalis nahe.

Patienten und Methodik: Es wurden 70 Biopsien von Patienten mit einer Arteriitis temporalis ohne histologische Bestätigung einer RZA (RZA-negativ) untersucht und mit 49 Biopsien aus Temporalarterien verstorbener Patienten ohne RZA verglichen. Zudem wurden 93 Biopsien von RZA-positiven Temporalarterien untersucht. Immunzytochemisch wurden die Häufigkeit von VZV-Antigen in Biopsien und anhand der Subtypisierung von Entzündungszellen das Ausmaß der Inflammation untersucht.

Ergebnisse: VZV-Antigen wurde bei $64 \%$ der RZA-negativen Temporalarterien von Patienten mit Verdacht auf RZA nachgewiesen. Bei RZA-positiven Temporalarterien war VZV-Antigen in $73 \%$ nachweisbar, bei normalen Temporalarterien nur in $22 \%$. Dabei war VZVAntigen vor allem in der Adventitia

Nagel MA, White T, Khmeleva $\mathrm{N}$ et al. Analysis of varicellazoster virus in temporal arteries biopsy positive and negative for giant cell arteritis. JAMA Neurol 2015; $72: 1281-7$ und in perineuralen, Claudin-1 exprimierenden Zellen zu finden.

Schlussfolgerungen: Bei Patienten mit klinisch wahrscheinlicher RZA lässt sich unabhängig von der RZA-Pathologie VZV-Antigen signifikant häufiger nachweisen als bei gesunden Kontrollen.

\section{- Kommentar von Matthias Maschke, Trier}

\section{RZA zukünftig antiviral behandeln?}

Diese Studie bestätigt, dass die Infektion mit VZV in der Pathogenese der Arteriitis temporalis eine Rolle spielt. Ergänzend zu der in Neurology veröffentlichten Studie von Gilden et al. lässt sich VZV-Antigen selbst dann nachweisen, wenn die Histologie keine RZA-typische Pathologie vorfindet. Inwieweit dies dann dazu führen könnte, dass demnächst auch antivirale Medikamente bei Patienten mit einer Riesenzellarteriitis zum Einsatz kommen, muss Gegenstand weiterer Studien sein.

\section{Referenzen:}

1. Nagel MA et al J Neurol Sci 2013; 335: $228-30$

2. Gilden D et al. Neurology 2015; 84: 1948-55

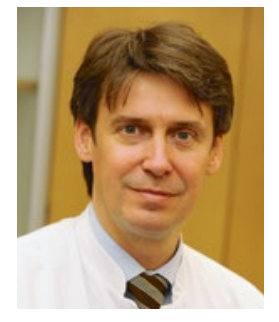

Prof. Dr. med. Matthias Maschke, Trier 